\title{
Your Primary Care Doctor May Have an MD from Cuba: Experiences from the Latin American Medical School
}

\section{Conner Gorry MA}

On the 40th anniversary of the Alma Ata Declaration that affirmed health for all a right and primary health care the route to guarantee that right, $\mathrm{WHO}$ and $\mathrm{PAHO}$ have issued a call to action to convert universal health into reality for the nearly four billion people worldwide lacking full coverage of essential health services. There is some urgency to this movement: WHO estimates the health workforce shortage of nearly 8 million could reach almost 13 million by 2035. And the USA is not exempt: medical associations and special commissions set up to investigate the shortage of primary care physicians, especially "under-represented minority" doctors, have issued report after report on this growing health care emergency. The biggest question looming is: where will the health care providers come from, especially well-trained primary care doctors who want to work for those who need them most?

Part of the answer may be found in Cuba and its Latin American School of Medicine (ELAM), which has graduated some 28,500 MDs from 103 countries since its first class of 2005-including nearly 200 from the USA. Graduates, most from low-income families, are not only admitted for their intellect and academic records, but also for their social commitment,

Conceived in 1998, ELAM provides six years of medical education for its students, including those from the USA. They are asked to commit to practice in shortage areas back home or in similar communities elsewhere. They receive full scholarships from Cuba, thus graduating free of the debt burden that might otherwise drive them into career paths that usually pay higher salaries. From postearthquake Haiti and Chicago's Cook County Jail, to Honduras' isolated Caribbean coast and Ohio's Transgender Primary Care Clinic, these young doctors are also offering an example to others of their generation.

The innovative program combines community-based, preventive care and a public health approach with clinical skills training. The curriculum incorporates basic sciences as well as clinic and hospital rotations in pediatrics, internal medicine, ob-gyn and other specialties, giving these future doctors practical experience with patients and group problem-solving throughout their medical studies. They also take courses in disaster medicine that include preparedness as well as response.

Today, a higher percentage of graduates from ELAM are working in disadvantaged US communities than those from any medical school in the USA itself, something that has attracted the attention of US medical educators, looking to see how they might increase their contribution to solving the physician shortage in these areas .

The 172 US doctors who have graduated from ELAM have experienced for themselves the hands-on clinical training, emphasis on teamwork, and integrative biopsychosocial approach underpinning Cuba's universal health system. And they have done so while living and studying in a foreign, resource-
Data Snapshot: US Students and Graduates of ELAM

\begin{tabular}{l|r}
\hline Indicator & \multicolumn{1}{|c}{ Value } \\
\hline Students currently enrolled & 72 \\
\hline Graduates & 172 \\
\hline Graduates in residency or post residency & 84 \\
\hline Graduates working in designated Health & $73 \%$ \\
Professional Shortage Area (HPSA) and/or & \\
Medically Underserved Area (MUA) & \\
\hline Specialty & Graduates n (\%) \\
\hline Family medicine & $49(58)$ \\
\hline Internal medicine & $21(25)$ \\
\hline Surgery & $5(6)$ \\
\hline Pediatrics & $4(5)$ \\
\hline Emergency medicine & $2(2)$ \\
Neurology & $1(1)$ \\
\hline Psychiatry & $1(1)$ \\
\hline Triple board & $1(1)$
\end{tabular}

*Those not working in an HPSA or MUA are working in the public sector with underserved communities.

Source: MEDICC data, April 2018, provided by Devon Baird, Director, Academic Programs

scarce country, often with no Spanish language skills upon arrival. The steep road to realizing their professional dreams doesn't end upon graduation, however: to practice back home, they must pass the three-step US Medical Licensing Examination (USMLE), plus secure and complete residencies.

MEDICC Review looked to a group of these US graduates to better understand their decision to study medicine in Cuba and how they faced the resulting challenges, as well as the relevance of their Cuban education to their current practice.

MEDICC Review: Studying medicine in Cuba is a bold-some would say radical-decision, especially given turbulent USCuba relations. What led you to apply to ELAM?

Parastou Malek (class of 2014): For me, studying at ELAM presented a unique opportunity to learn alongside a very diverse student body. Furthermore, I was desperate to find an effective way to gain the skills I needed to help my community and I knew learning Spanish would allow me to reach a much larger patient population. Previous ELAM graduates also talked about extremely supportive professors who created a friendly and encouraging learning environment for their students. I knew that I had the potential to thrive in such a setting and wanted to experience the difference between the Cuban and US education systems.

Melissa Davis (class of 2011): I believe health care is a human right and that physicians are obliged to bear witness to injustice and advocate for vulnerable populations; I was excited at the opportunity to join other young people with similar aspirations and beliefs. I was also motivated to learn medicine in a relatively resource-poor country so I could apply my knowledge in contexts 




Dr Parastou Malek (r) at ELAM's 2014 graduation ceremonies.

besides modern US health care settings. I knew attending ELAM would enable me to practice wherever I wanted in the future without being held back by debt.

Pasha Jackson (class of 2015): Before coming to Cuba, I was a professional player in the National Football League (NFL). Stigmatized by injuries, I floundered from team to team and found my core values of brotherhood, collaborative work and group excellence were drowning-as was I. Inspired by Cuba's examples of global humanitarianism, ELAM fueled my exit from the NFL with renewed hopes for meaningful work within a team environment. In short, ELAM appealed to my altruistic side. I was also excited to refocus on academic interests that were deemed incompatible for high-level athletes.

Rose Lafleur (class of 2009): When I began applying for medical school and interacting with other prospective medical students, I became unsure about my career path-for the first time

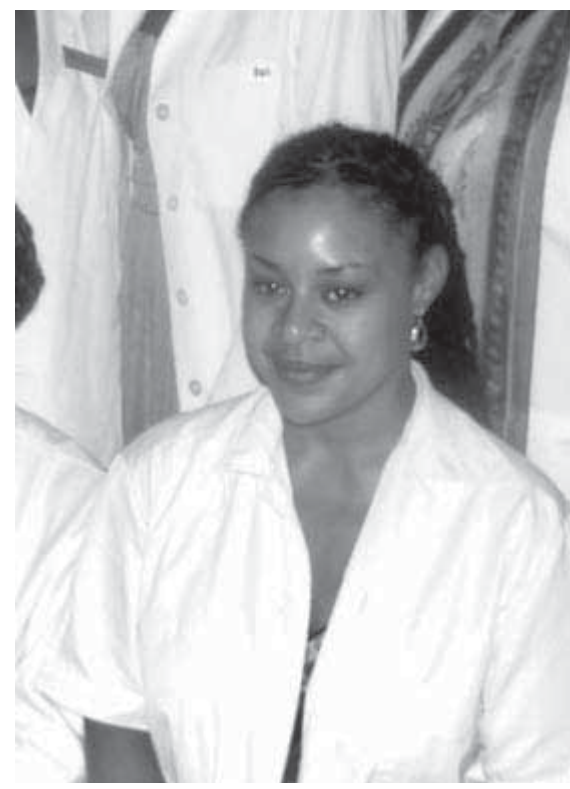

Dr Rose Lafleur in her student years at ELAM. which schools were most illustrious, bothered me. I didn't hear anything about what motivates me: working with people, the healing aspects of medicine, or the influence a doctor can have on their community. I felt isolated. And sad. Then I heard about ELAM.

When I requested a brochure, I received a piece of trifolded paper in the mail. On the cover was a picture of a Cuban doctor sitting in the front yard of a farm home, with a couple of chickens around him, auscultating a patient. The brochure contained the basic sciences curriculum and a few words about ELAM's mission. That simple, humble imagery, along with the emphasis on community-oriented health, convinced me that I would find my path in Cuba.

Sarah Hernández (class of 2016): I first heard about Cuba's international medical school during a ten-week University of California study abroad program in Havana. One of my Cuban professors explained how ELAM works and I was instantly attracted to the idea of being trained as a physician in a system that considers health a human right. I felt a tangible excitement, a flame ignite inside of me, when he took me to visit the ELAM campus. Immediately I knew I wanted to study there. ELAM was my first-and only-choice for medical school. I was secure in my commitment to study medicine within a health care system that provides equal access to its entire population.

MEDICC Review: Cuba can be a difficult context in which to live and study. Can you describe some of the challenges or culture shock you faced upon arrival?

Pasha Jackson: For me, it was a shock living with students largely in their teens. [most students arrive at ELAM out of high school-Eds.] I had already completed my undergraduate degree and played professional football for three years when I started at ELAM. Once I moved from the dorms into the community [All ELAM students spend the first two years on campus and then move to neighborhoods for their clinical years- Eds.], I found an interesting juxtaposition between the foreign and familiar. Like Oakland, where I'm from, these were people who were resource poor, but culturally and spiritually rich. This fosters a special type of ingenuity that, for me, represented hope. The real culture shock for me, however, was to be among poor people who were educated, had great access to health care, were free of drug epidemics and most impressive of all, relatively nonviolent.

Parastou Malek: I absolutely experienced culture shock, especially since I lived through a hurricane in my first few months in Cuba. When I arrived in 2008, there was barely Internet, Cubans didn't have cell phones, they didn't own businesses. There weren't that many restaurants and little to buy. The country changed a lot while I was there, but it was still an adjustment.
Sarpoma Sefa-Boakye (class of 2009): Having my first female science professor of African descent like me and the fact that most of my professors looked like me was shocking. I had just finished premedicine at UCLA where I was always the minority; I was actually told by my very first science teacher as a child that I would never make it as a doctor.

Sarah Hernández: My first night I just sat on my bed, silently, looking around blankly, trying to process what was to come. I think the biggest adaptation was the lack of utilitarian items. I longed for more stuff, but it was just stuff I realized, and the Cubans around me were living loving, functional lives with so much less. I was humbled, because I could always obtain necessities-a small care package or by asking someone to bring me something.

MEDICC Review: What about studying entirely in Spanish? Did that present difficulties? 


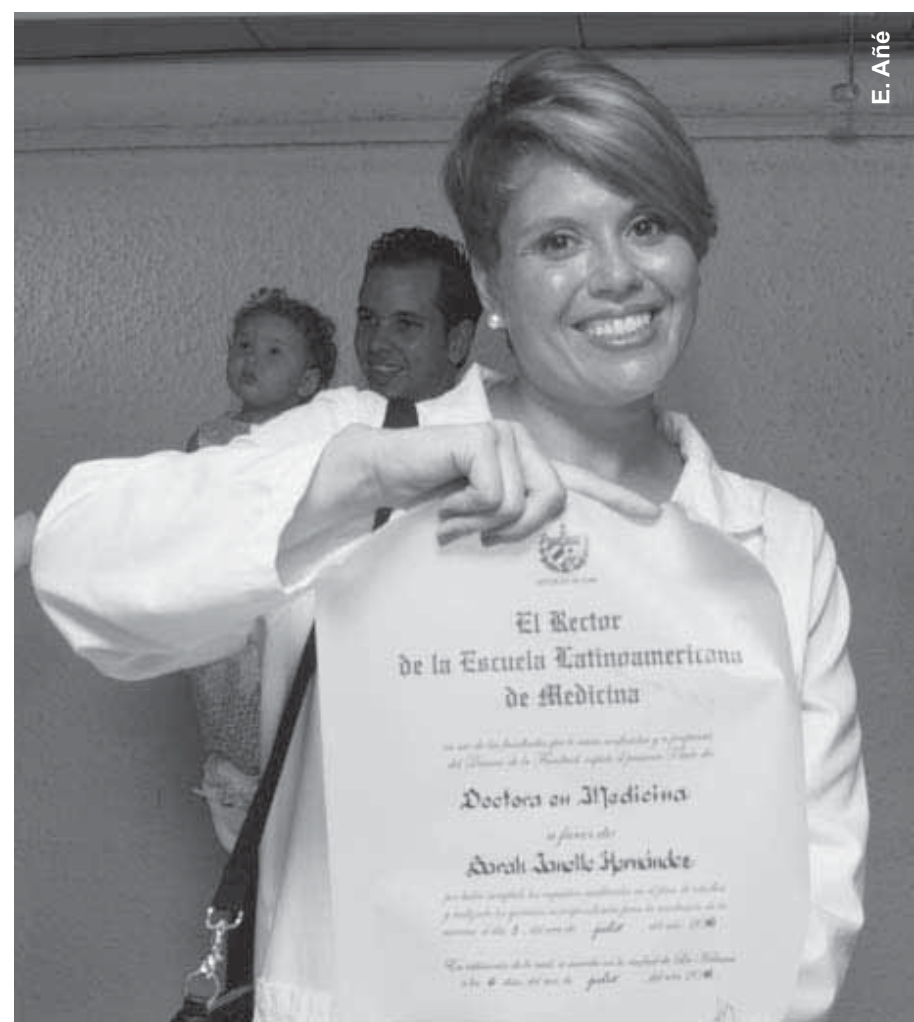

Dr Sarah Hernández on graduation day, 2016.

Sarah Hernández: Although I'm of Mexican-American descent, I didn't learn Spanish as a child and arrived in Cuba with the little I learned in high school. Being immersed in a Spanish-speaking country and medical school, with so many different accents, was mind blowing. Some of my colleagues in the USA whose first language is Spanish aren't fluent in medical Spanish; my ability to speak conversational and medical Spanish is such an asset in this context.

Rose Lafleur: I didn't speak a word of Spanish when I arrived in Cuba. And it was hard for me; it took me about two years to speak intelligibly-this made me very shy about trying. I only began communicating well when clinical rotations began in our third year and I had to speak with patients and communicate information to my attending professors.

Pasha Jackson: I spoke very little Spanish when I applied to ELAM. After an evaluation, I was placed in a six-month Spanish immersion course at the school. I was concerned I wasn't learning fast enough but one of my Spanish professors told me it takes roughly five years of dedicated study to become fluent in a language. Indeed, by my fifth year at ELAM, I was freely and independently traveling throughout Havana, engaging in deep, thoughtful conversations, and even giving speeches in Spanish. Increasing my Spanish fluency was challenging, but manageable, thanks to the school's diverse and approachable student body.

Parastou Malek: I only knew very basic Spanish when I arrived, but it came to me a lot easier than the four previous languages I'd learned. I requested a dorm with Latin American students and roomed for two years with students from Uruguay. I also made a huge effort to make friendships outside of the US delegation. I believe my Spanish reached the level it did thanks to the help I received from my Spanish-speaking classmates. Some used to spend hours tutoring me and helping me understand lectures.

\section{MEDICC Review: What was the medical training in Cuba like? Are there specific elements or experiences that stick with you?}

Melissa Davis: In third year we began clinical rotations and I wondered how Cuban patients would respond to me as an American. I was surprised at how generous, welcoming and kind they were. I remember once in the hospital, a patient called from across the hall to show a group of us a wound. He wasn't our patient and already had his diagnosis and treatment plan, but he wanted to contribute to our education by showing us his skin lesion.

As third-year students, we were an integral part of the care team and afforded the same respect as doctors. We were never treated as a burden or made to feel like what we did wasn't useful. This made me want to work even harder. Everything we wrote in a patient's clinical record became official after it was signed off by our attending. As an intern in Cuba, I had high expectations both to learn and to teach the students a few years behind me. I didn't realize how formative all of these experiences were at the time, but now when I'm with my students in the USA, I try to make them a part of the team. It's important that they realize they're due the same respect as their superiors-but I also help them understand that with respect comes great responsibility, too.

Rose Lafleur: My medical education and years in Cuba provided me with a deep understanding of the importance of primary care and health education, plus the responsibility patients have for their

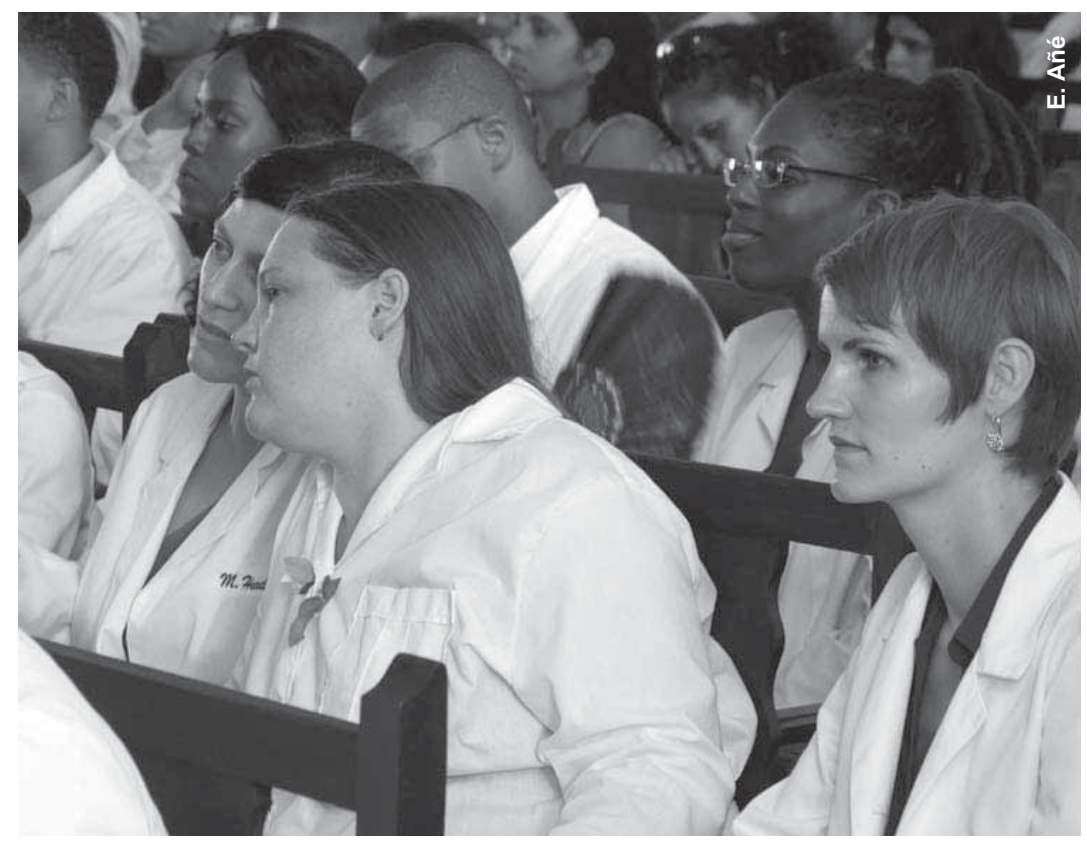

Dr Melissa Davis (r) with other US classmates at graduation 2011. 
individual health. In fourth year, I did the family medicine rotation and began to truly understand the role of the family doctor. In my practice today, I spend time explaining each patient's condition, detailing where they can look for more information and orienting them about possible complications and how to avoid them.

Coming from the Cuban medical setting where health education is directly linked to outcomes motivated me to start a small nonprofit in Oakland, California, training young people to become health educators. The aim is to provide these young people with employable skills and improve community health outcomes.

Heather Krause (class of 2015): My career in medicine started nearly a decade ago when I trained to be an emergency medical technician in my hometown of Rockport, Texas. Working in disaster medicine is a dream l've nurtured for a long time and the education I received at ELAM prepared me to work in a disaster setting with limited resources. In January 2018, I put my experience and knowledge to use when I joined the New York State Nurses Association medical mission to Puerto Rico.

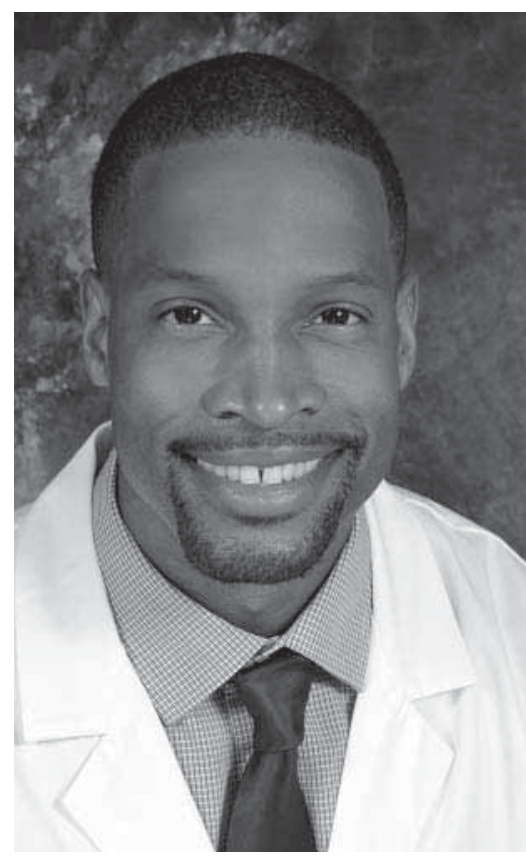

Dr Pasha Jackson, family medicine resident, Ponoma (CA).
Pasha Jackson: Training in Cuba contributed greatly to my passion for incorporating social determinants into the dominant biomedical culture in the USA. My ELAM education helped reveal the degree to which biological, psychological and social spheres of health are inseparable. Since starting my residency program, l've received very consistent feedback from superiors applauding the exploration of my patients' social factors. Cuba's well-known resourcefulness inspires me to explore beyond popular paradigms for integrative solutions to health and wellness. For example, it's quite common for me to coach breathing techniques for anxiety and stress disorders. Often my recommendation of alternative treatments gives my patients a greater sense of confidence in my role as their doctor, strengthening our overall relationship. My time studying in Cuba made me believe in the power of relationship-based medicine, which remains one of my top daily goals and reliable clinical tools.

Parastou Malek: Practicing here in the USA, I do my best to always look at my patients from a biopsychosocial perspective, trying to understand where they are coming from and how I can meet their needs as a physician and as a responsible member of their community. I try to use every opportunity to create good rapport with my patients and gain their trust. I have been taking motivational interviewing training courses in order to find efficient and effective ways to counsel my patients on making lifestyle

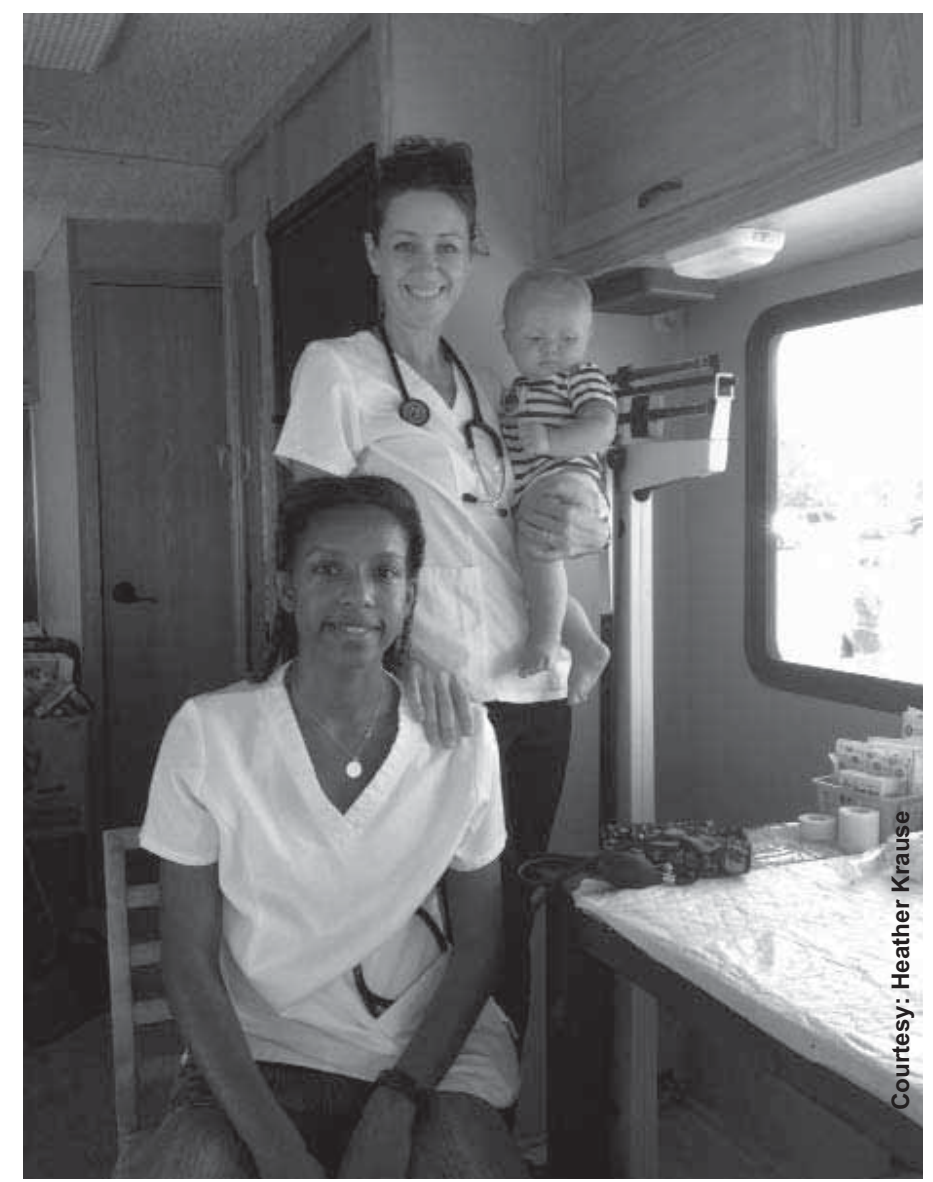

Dr Heather Krause (top) with Dr Mercedes Charles, 2017 ELAM graduate

changes-smoking cessation, diet modification and how to incorporate more exercise, meditation and yoga into their daily routine. Coming from a Cuban context, I try to be as resourceful as possible and I'm mindful of not wasting resources.

MEDICC Review: Can you describe what it was like returning to the USA as a foreign medical graduate and navigating the USMLEs and process for acceptance into a medical residency program?

Rose Lafleur: I had to re-establish myself completely as a productive, rent-paying individual in a new city. The first year was extremely difficult; I struggled with job security, as well as studying and paying for exams. It wasn't a linear trajectory—but eventually I matched into an internal medicine residency.

Melissa Davis: The USMLE Step 1 exam was the hardest part for me. The Cuban curriculum is designed for group problem solving and relating the concepts directly to our future practice, while the Step 1 questions were more obscure and less clinically relevant. I studied hours a day for several months to pass the exam. Upon starting a family medicine residency, I had to get used to the inordinate number of prescription medication choices and restrictions based on insurance coverage and cost. An even bigger obstacle that still saddens me is the distrust and suspicion directed toward the medical establishment that I sometimes see here but did not experience in Cuba. When people have a negative 


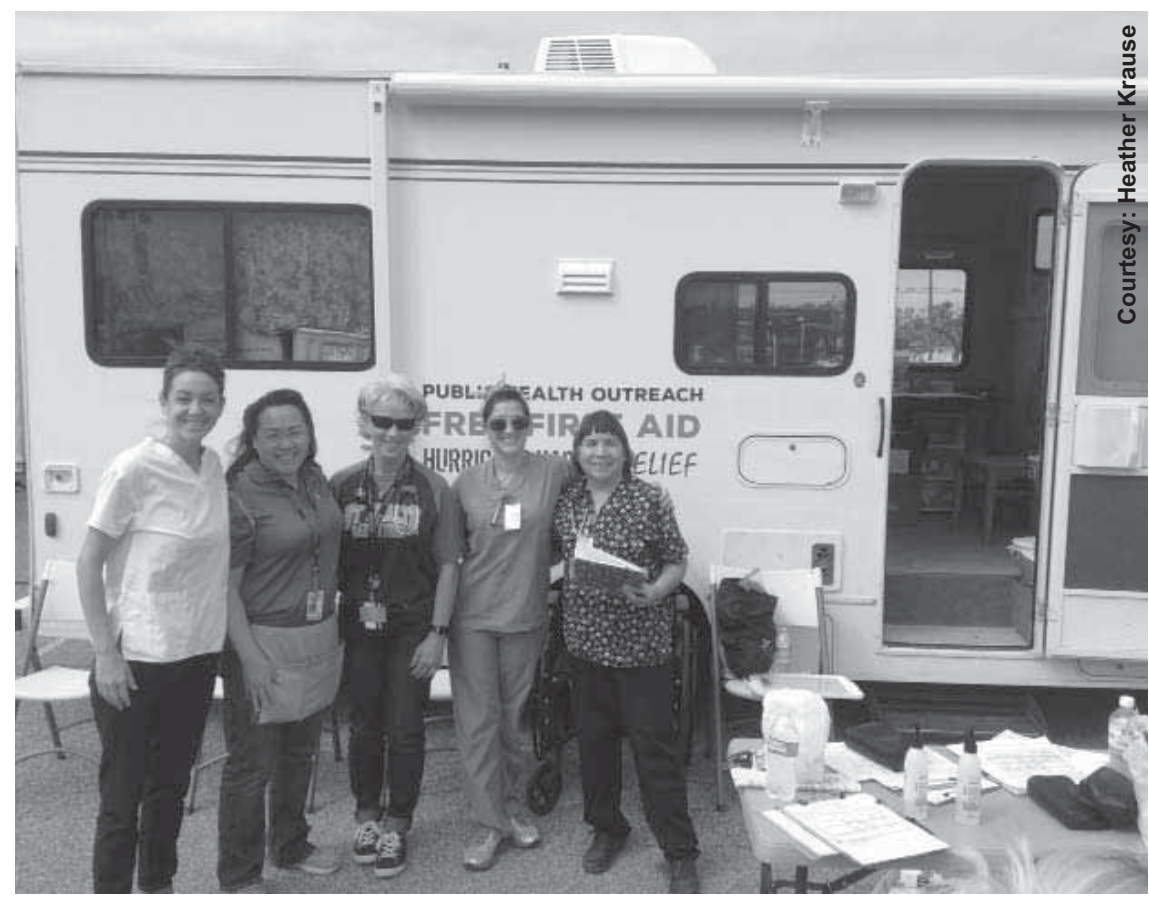

Rockport Strong Mobile Medical Unit, Hurricane Harvey disaster relief. into medicine as well as a steady, living wage before I started residency in July 2017.

Parastou Malek: There were so many challenges upon my return-learning how to study for the USMLEs, for example, and then passing them, plus honing my interview skills and connecting with relevant medical programs-it was all really difficult. MEDICC provided endless support through its various programs for ELAM graduates and I don't know where I would be were it not for that. The long hours we're expected to work as residents are especially tough, as is making peace with the broken US health system. On a daily basis I see how the system here can work against patients instead of for them. It is very discouraging and disheartening.

\section{MEDICC Review: Are you practicing or in residency now?}

Heather Krause: When Hurricane Harvey hit my hometown in August 2017, my husband and I evacuated in our 24-foot mobile home. As soon as the storm passed, we converted experience with a doctor or in a health care setting, it can be very hard for them to come back. As a result, their chronic conditions go uncontrolled and we see complications which could have been prevented if they'd had access to a trusted family physician. In Cuba the doctors are very much a part of the community and are sought out for advice and guidance.

Sarpoma Sefa-Boakye: Returning home was especially hard for me. I thought the hardest part was going to be taking the USMLEs and getting into residency, but the most difficult part was learning how to work in the US health care system. I did three years of family medicine residency on the US-Mexican border in San Diego and it was hard advocating for my patients in hospitals with very few resources for underinsured patients. Cuba clearly didn't prepare us for that.

Sarah Hernández: Due to the way the Cuban academic calendar is structured, many US graduates have a gap year after graduation, the ten months postgraduation/preresidency. For me personally, it was very difficult socially, psychologically, and especially financially. I went from living in my own apartment back to living with my mother-who graciously took me in, along with my Cuban husband, and our two-year-old son. It was hard to get steady work, since I needed time for the three-month residency interview process, so I took odd jobs, including scanning documents at a law firm; on weekends, I washed dishes at a bar, earning the nickname "Doctor Dishwasher." I even donated bone marrow, white blood cells and blood to cover living expenses and pay for my Step 3 Exam. I was fortunate enough to do a research fellowship at Highland Hospital (in Oakland, California) for three months, which gave me a wonderful transition back

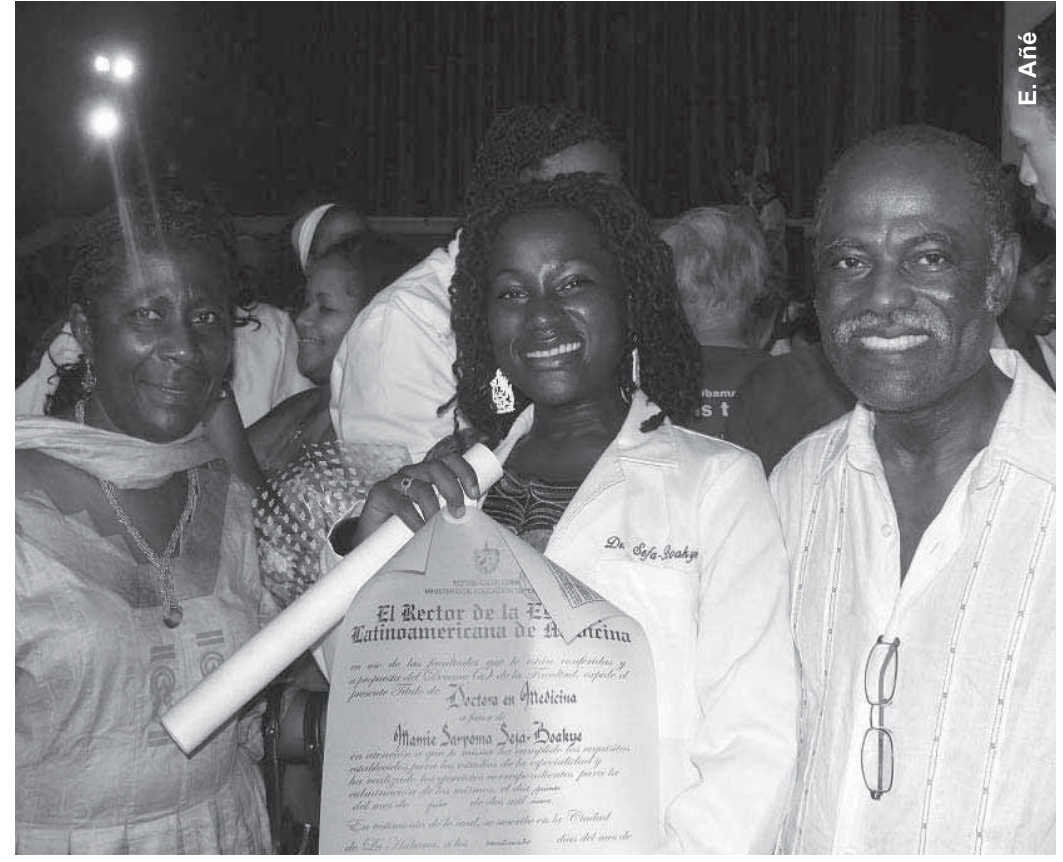

Dr Sarpoma Sefa-Boakye with her parents at graduation in Havana, 2009. 
(we saw several patients who lost their insurance plans after the storm) and/or closure of health facilities due to storm damage.

We collected public health data about the patients we treatedthe survey was processed by the University of Nebraska Medical Center-and found: $61.5 \%$ reported not having a primary care physician; $68 \%$ didn't have health insurance; $34 \%$ had used the emergency room for primary care services; $73.6 \%$ reported their health was negatively impacted by the hurricane; $38 \%$ lost their home to hurricane damage; $49.3 \%$ had a diagnosed medical condition; $35.7 \%$ owed money to a hospital for medical bills. Right now I'm pursuing a master's degree in public health (MPH) at George Washington University's Milken Institute of Public Health.

Sarah Hernández: I am currently a first-year resident at Pomona Valley Hospital Medical Center in Pomona, California, participating in the family medicine residency program. While I'm not exactly sure what my plans are after residency, I am committed to working in diverse, underserved communities. I also foresee myself working with adolescents.

Parastou Malek: At this point, I see myself doing outpatient primary care, mostly. Right now, I'm at the University of New Mexico Community and Family Medicine residency program. I have a variety of interests that I want to explore over the next several years to see what best fits. Stay tuned!

Melissa Davis: I am a clinical assistant professor of Family Medicine at Ohio State University (OSU) and care for a diversity of patients in inner city Columbus. I have some other roles, too. I'm a Teen Clinic physician at the Nationwide Children's Hospital Hilltop and Near East Side Primary Care Centers. In addition to urban family medicine, I have developed a special interest in providing high-quality primary care for transgender or gender nonconforming individuals and see patients weekly at the Transgender Primary Care Clinic at OSU, where we provide comprehensive primary care, including hormone therapy. I enjoy the opportunities here to learn from and teach young health professionals and I also spend six weeks a year supervising residents in the family medicine teaching service. By this summer, I will have obtained my MPH from OSU College of Public Health.

Rose Lafleur: I plan to work as a primary care doctor in a community setting but am also considering ways to insert myself

\section{For more information about ELAM:}

Giraldo G. Cuba's piece in the global health workforce puzzle. MEDICC Rev. 2007 Oct;9(1):44-7.

Gorry C. Cuba's Latin American Medical School: Can socially accountable medical education make a difference? MEDICC Rev 2012 Jul;14(3):5-11.

Gorry C. Latin American Medical School Class of 2015: Exclusive with Cuban-trained US graduates. MEDICC Rev. 2015 Jul;17(3):7-11.

Morales Suárez IR, Fernández Sacasa JA, Durán García F. Cuban Medical Education: Aiming for the Six-Star Doctor. MEDICC Rev. 2008 Oct;10(4):5-9.

Neusy AJ, Palsdottir B. A roundtable of innovative leaders in medical education. MEDICC Rev. 2008 Oct;10(4):20-4.

into global public health-thinking globally and acting locally is definitely in my future. At present I'm a first-year resident at Wyckoff Heights Medical Center, a small community hospital in Brooklyn, NY.

Sarpoma Sefa-Boakye: I work as a family medicine physician at a primary care clinic in San Diego county, while also serving as International Medical Director of the Birthing Project USA, which aims to enhance health outcomes for women of color primarily. My goal is to continue promoting the Cuban primary care model through my work both internationally and domestically.

Pasha Jackson: I'm Co-Chief Resident at Pomona Valley Hospital Medical Center's Family Medicine Residency Program in Pomona, California. As for my future plans-I'll have to get back to you on that one! $-1 /$ -

Disclosure: MEDICC Review is published by MEDICC, which provides mentors, other guidance and financial support to US ELAM graduates to defray costs of USMLE and preparatory courses. 